\title{
Managing the Newsvendor Modeled Product System with Random Capacity and Capacity-Dependent Price
}

\author{
Qingying Li, ${ }^{1}$ Ciwei Dong, ${ }^{2}$ and Ruixin Zhuang ${ }^{3}$ \\ ${ }^{1}$ Glorious Sun School of Business and Management, Donghua University, 1882 West Yanian Road, Shanghai 200051, China \\ ${ }^{2}$ School of Business Administration and Center for Industrial Economy, Zhongnan University of Economics and Law, \\ Wuhan 430073, China \\ ${ }^{3}$ China Foreign Exchange Trade System, Building 30, 1387 Zhangdong Road, Shanghai 201203, China
}

Correspondence should be addressed to Ciwei Dong; dongciwei@znufe.edu.cn

Received 25 September 2014; Revised 12 December 2014; Accepted 13 December 2014

Academic Editor: Tsan-Ming Choi

Copyright (C) 2015 Qingying Li et al. This is an open access article distributed under the Creative Commons Attribution License, which permits unrestricted use, distribution, and reproduction in any medium, provided the original work is properly cited.

\begin{abstract}
We consider a newsvendor modeled product system, where the firm provides products to the market. The supply capacity of the product is random, so the firm receives either the amount of order quantity or the realized capacity, whichever is smaller. The market price is capacity dependent. We consider two types of production cost structures: the procurement case and the in-house production case. The firm pays for the received quantity in the former case and for the ordered quantity in the latter case. We obtain the optimal order quantities for both cases. Comparing with the traditional newsvendor model, we find that the optimal order quantity in both the procurement case and the in-house production case are no greater than that in the traditional newsvendor model with a fixed selling price. We also find that the optimal order quantity for the procurement case is greater than that for the in-house production case. Numerical study is conducted to investigate the sensitivity of the optimal solution versus the distribution of the random capacity/demand.
\end{abstract}

\section{Introduction}

Newsvendor molded product system, where the firm needs to decide how many products to order for a market with uncertain demand, is very common in our real life, and the related problems are wildly studied in the literature. Interested readers may refer to Khouja [1] and Qin et al. [2] for various newsvendor problems. In the traditional newsvendor model, the market prices of the products are usually exogenously given and are not affected by other factors. However, in fact the market price might be affected by some external factors, such as supply capacity. For example, the market prices of import products by the oversea procurement service might be increased, if the custom sharply controls the import products. In this example, the custom control is a kind of supply uncertainty for the oversea procurement service, which causes a random capacity for the business and affects the market price. Thus, the characteristic of capacitydependent price cannot be neglected when we study the problems for the newsvendor modeled product system.
On the other hand, in addition to the demand uncertainty, supply uncertainty also universally exists in the practical situations. There are three common types of supply uncertainty in supply quantity in the literature. The first one is the random yield, where the quantity received is not the same as the quantity ordered and is usually the fraction of the quantity ordered. The second one is the supply disruption, where a supplier may not be available to provide the service for random durations. Usually, all ordered items will be delivered if the supplier is available; and nothing will be delivered if the supplier is unavailable. The third one is the random capacity, where the supplier's capacity is a random variable [3]. Interested readers may refer to Yano and Lee [4], Tajbakhsh et al. [3], and Snyder et al. [5] for a summary of the supply uncertainty issues on the production/inventory problems. To effectively match the supply with demand, the system manager needs to make decisions with considering supply uncertainties.

Motivated by the phenomenon that the two characteristics mentioned above, that is, capacity-dependent price and 
supply uncertainty, wildly exist in the practical situations, in this paper we study a newsvendor modeled product system with the consideration of these two characteristics. Specifically, we consider that the product provider faces random capacity, and the market price of the product is capacity dependent. We do not make any particular requirement on the price, except that it decreases in the supply capacity. Regarding the production cost, we consider two types of structures. The first one is the in-house production case, where the production cost depends on the quantity ordered. The second one is the procurement case, where the production cost depends on the quantity received. Both cases are common in practical situations, depending on whether the product provider is a manufacturer or a retailer.

As far as we are concerned, this is the first work considering random capacity, capacity-dependent price, and the two cost structures. We investigate the optimal solutions under each cost structure. We find that, in the procurement case, the expected profit is quasi-concave, and, in the in-house production case, the expected profit is concave. Thus, we obtain the unique optimal solutions for both cases. Then, we compare the optimal solutions under the two cost structures with that in the traditional newsvendor model. We find that, in each case, the optimal order quantity is no greater than the optimal quantity in the traditional newsvendor model with a fixed selling price. We also find that the optimal solution for the procurement case is no smaller than that for the in-house production case. Numerical studies are also conducted to investigate the sensitivities of the optimal solutions versus the distribution of the random capacity. Via some numerical tests using normal distributed random capacity and demand and linear price function, we observe that the optimal quantities decrease in the deviation of the random capacity in both cases, and the optimal quantity of the in-house production case is more sensitive in the deviation of the random capacity.

The rest of the paper is organized as follows. In Section 2, we review the related literature. In Section 3, we introduce the model and solve two models in two subsections. Section 4 presents the numerical study results, and Section 5 concludes the paper. All the proofs are regulated to the Appendix.

\section{Literature Review}

Our work is related to the two streams of the research in the operations management/operations research literature. The first stream is literature on the supply uncertainty, and the second stream is literature on the production planning problem with variable market price.

Supply uncertainty has received extensive attention in the literature. There are three types of commonly seen uncertainties in the supply quantities: random yield, supply disruption, and random capacity [3]. Yano and Lee [4] provide a comprehensive review for the random yield. More recent works in the random yield include Gurnani et al. [6], Tomlin and Wang [7], Dada et al. [8], Giri [9], Tang and Kouvelis [10], and Chao et al. [11]. Many literatures on supply disruption consider the single supplier thus focusing on using the inventory policy to handle the supply uncertainty. Examples of such studies can be found in Meyer et al. [12], Bielecki and Kumar [13], Parlar and Berkin [14], Parlar and Perry [15], Parlar [16], Moinzadeh and Aggarwal [17], and Arreola-Risa and DeCroix [18]. Recently, there are some studies on supply disruption considering the multiple suppliers and focusing on souring mitigation. Examples of such studies can be found in Tomlin [19], Chopra et al. [20], Li et al. [21], Gupta et al. [22], and Ray and Jenamani [23]. Comprehensive review of the literature on supply disruption can be found in Snyder et al. [5].

As we consider the random capacity in this paper, we next restrict our attention to the studies considering random capacity. Arrow et al. [24] may be considered as the first work on this topic, where they assume that the amount received is independent of the order quantity but depends on a certain probability distribution. Ciarallo et al. [25] investigate a multi-period production planning problem with the random capacity. They show that, for the single-period model, the random capacity will only affect the optimal expected profit but not affect the optimal order quantity. They then extend the analysis and show that an order-up-to policy, which depends on the capacity distribution, is optimal for the multiperiod and infinite-horizon problem. Wang and Gerchak [26] consider an extended model by allowing both random yield and variable capacity. A similar model with dependent demand and supply is studied by Okyay et al. [27]. Wu et al. [28] investigate a risk-averse newsvendor model with random capacity, where the risk criteria VaR and CVaR are considered. There are also some studies considering random capacity without using newsvendor setting. For example, Bollapragada et al. [29] consider an assembly system where the component providers face the random capacity problems, and Erdem et al. [30] study an EOQ model where there are multiple suppliers facing the random capacity problems. Other studies that consider the random capacity include Khang and Fujiwara [31], Kouvelis and Milner [32], and Bollapragada et al. [33]. In all of the works about the random capacity mentioned above, the market selling price is fixed. In our work, we let the market price be dependent on the random capacity.

The variable price is also a common issue in the production planning problems, which covers a huge literature. Here, we only review those under the uncertain supply circumstance. Kazaz [34] investigates the production planning problem for olive oil with random yield rate, where both the cost and the price are dependent on the yield rate. His work is extended by Kazaz and Webster [35] by allowing the demand be yield rate dependent and considering risk-averse objective. Similar to the settings on the price in Kazaz [34] and Kazaz and Webster [35], we consider the capacity-dependent price, but we focus on random capacity. Tang and Yin [36] study the pricing policy for a system with both uncertain supply and demand, where the demand is price dependent. $\mathrm{Xu}$ and $\mathrm{Lu}$ [37] consider a price-setting newsvendor problem where the manager needs to make a joint decision on the inventory and pricing. They consider two production cost structures and compare the optimal solutions for the two cases. Li and Zheng [38], and Chao et al. [11] also investigate the joint decision of the pricing and inventory policies with random yield. Although we adopt the two cost structures used in $\mathrm{Xu}$ and $\mathrm{Lu}$ 
[37], our models are fundamentally different: (i) we consider a capacity-dependent market price and they consider a fixed market price, (ii) we consider random capacity and they focus on random yield rate, and (iii) they consider price-dependent demand and we assume demand is independent of the price or the capacity.

\section{The Model}

We consider a newsvendor modeled product system, where a firm provides products to the market. Before the selling season, the firm makes an ordering decision, say $q$ units. The supply is unreliable. Specifically, we consider a random capacity, denoted as $u$. Let $u$ be the random supply capacity, and let $f(\cdot)$ and $F(\cdot)$ be its $p d f$ and $c d f$, respectively. Then, the received quantity is the minimum value between the order quantity and the supply capacity, that is, $\min \{q, u\}$. Regarding firm's cost for purchasing these $\min \{u, q\}$ units, we consider two cases. The first one is the in-house production case, where the firm needs to pay for the quantity ordered, that is, $q$. This case is common when the firm plays a role as a manufacturer. The second one is the procurement case, where the firm pays for the quantity received, that is, $\min \{q, u\}$. This case is common when the firm plays a role as a retailer. Let $c$ be the unit cost for the products.

In the selling season, the market price depends on the supply capacity. Let the market price be $p(u)$. We only require that $p(u)$ satisfies the following two properties. (i) $p(u)$ decreases in $u$; that is, the market price decreases with supply capacity. (ii) $p(u) \geq c$ for any $u$; that is, the market price is no less than the unit production cost, because the firm will face nonprofitable market price otherwise. Note that, in our analysis, we will use "decrease" and "increase" to represent "non-decrease" and "non-increase" for simplicity.

We let $D$ be the demand, which is a random variable independent of the capacity $u$. Let $G(\cdot)$ and $g(\cdot)$ be its $c d f$ and $p d f$, respectively. We do not have any specific requirement on the demand $D$. We assume the unmet demand is lost, and the unit salvage value of the leftover products is $s$, where $s<c$. For notational convenience, we also let $\bar{F}(x)=1-F(x)$ and $\bar{G}(x)=1-G(x)$ (see Notations).

We first give some preliminaries about the traditional newsvendor model. Let $\pi(q)$ be the expected profit in the traditional newsvendor model, given that the order quantity is $q$, and let $q_{0}^{*}$ be the optimal order quantity. Then, the firm's profit is given by

$$
\pi_{0}(q)=(p-s)\left[q-\int_{0}^{q} G(\xi) d \xi\right]-(c-s) q,
$$

which is concave, and the optimal order quantity satisfies

$$
\bar{G}\left(q_{0}^{*}\right)=\frac{c-s}{p-s} .
$$

(See, e.g., Zipkin [39].) Note that, in (1), we let $p$ be the constant selling price under the traditional setting. Next, we proceed to our random-capacity-dependent models with the two types of production cost.
3.1. The Procurement Case. We first consider the procurement case, where the firm pays for the received quantity. Let $\pi_{\mathrm{pc}}(u, q)$ be the firm's profit given that the supply capacity is $u$ and the order quantity is $q$. Then, $\pi_{\mathrm{pc}}(u, q)$ differs from $\pi_{0}(\min \{q, u\})$ only in the fact that the market price $p(u)$ is capacity dependent rather than a constant. Replacing " $p$ " in (1) by “p(u)," we have

$$
\begin{aligned}
& \pi_{\mathrm{pc}}(u, q) \\
& \quad= \begin{cases}(p(u)-s)\left[u-\int_{0}^{u} G(\xi) d \xi\right]-(c-s) u, & \text { if } u<q ; \\
(p(u)-s)\left[q-\int_{0}^{q} G(\xi) d \xi\right]-(c-s) q, & \text { if } u \geq q .\end{cases}
\end{aligned}
$$

The expected profit can then be obtained by taking expectation on $\pi_{\mathrm{pc}}(u, q)$ over $u$. Denote $\Pi_{\mathrm{pc}}(q)$ as the expected profit for the procurement case. We have

$$
\Pi_{\mathrm{pc}}(q)=\int_{0}^{+\infty} \pi_{\mathrm{pc}}(u, q) f(u) d u .
$$

Consider $\pi_{\mathrm{pc}}(u, q)$. For any given $u$ such that $u<q, \pi_{\mathrm{pc}}(u, q)$ is independent of $q$; and for any given $u$ such that $u \geq q$, $\pi_{\mathrm{pc}}(u, q)$ is identical to the profit for a traditional newsvendor model and is concave in $q$. Then, the integration $\Pi_{p c}(q)$ need not necessarily be concave in $q$. However, it can be shown that $\Pi_{\mathrm{pc}}(q)$ is quasi-concave in $q$ such that the optimal order quantity can still be uniquely determined, as summarized in the following proposition.

Proposition 1. Consider the procurement case. (i) The expected profit $\Pi_{p c}(q)$ is quasi-concave and (ii) the optimal order quantity, denoted as $q_{p c}^{*}$, is unique and satisfies

$$
\bar{G}\left(q_{p c}^{*}\right)=\frac{(c-s) \bar{F}\left(q_{p c}^{*}\right)}{\int_{q_{p c}^{*}}^{+\infty} p(u) f(u) d u-s \bar{F}\left(q_{p c}^{*}\right)} .
$$

Proposition 1 shows the optimal order quantity for the procurement case. Compare (2) with (5); we can find some similarities between the two expressions. In fact, we can establish the relationship between $q_{\mathrm{pc}}^{*}$ and the optimal order quantity in some traditional newsvendor model.

Let $q_{0}^{*}(x)$ be the optimal order quantity in the traditional newsvendor model given that the market price is $x$ (the traditional newsvendor model considers fixed market price). Then, we have the following proposition.

Proposition 2. Consider the procurement case. (i) The optimal order quantity for the procurement case $q_{p c}^{*}$ is no greater than the optimal order quantity for the traditional newsvendor model with fixed market price $p\left(q_{p c}^{*}\right)$, that is,

$$
q_{p c}^{*} \leq q_{0}^{*}\left(p\left(q_{p c}^{*}\right)\right)
$$

and (ii) the equality holds if and only if the market price $p(\cdot)$ is a constant. 
In the procurement model or the traditional newsvendor model, the firm pays for the received quantity. The difference between these two models are the random capacity and the capacity-dependent price. Ciarallo et al. [25] have shown that, in the traditional newsvendor model with fixed market price, the random capacity does not affect the optimal order quantity. This is consistent with part (ii) of Proposition 2. However, part (i) of Proposition 2 shows that this is not valid when the market price is also capacity dependent. In the procurement model, where the market price varies with the random capacity, the optimal order quantity $q_{\mathrm{pc}}^{*}$ is no greater than the optimal order quantity in the traditional newsvendor model with fixed market price $p\left(q_{\mathrm{pc}}^{*}\right)$.

3.2. The In-House Production Case. In this section, we consider the in-house production case, where the firm needs to pay for the quantity ordered, no matter whether the order cannot be fully received. Similar to the procurement case, we let $\pi_{\text {in }}(u, q)$ be the firm's profit when the capacity is $u$ and the order quantity is $q$. When the supply capacity is $u$, the market price is $p(u)$. In this case, the firm always needs to pay for the quantity ordered, that is, the quantity $q$. Similar to (3), we have

$$
\begin{aligned}
& \pi_{\text {in }}(u, q) \\
& \quad= \begin{cases}(p(u)-s)\left[u-\int_{0}^{u} G(\xi) d \xi\right]-(c-s) q, & \text { if } u<q ; \\
(p(u)-s)\left[q-\int_{0}^{q} G(\xi) d \xi\right]-(c-s) q, & \text { if } u \geq q .\end{cases}
\end{aligned}
$$

Let $\Pi_{\text {in }}(q)$ be the expected profit for the firm with in-house production cost. Then,

$$
\Pi_{\text {in }}(q)=\int_{0}^{+\infty} \pi_{\text {in }}(u, q) f(u) d u .
$$

Consider $\pi_{\text {in }}(u, q)$ given by (7). In the first case of (7), where $u<q$, the order quantity cannot be fully received such that the revenue from selling the products is independent of $q$, but the firm still needs to pay for the $q$ units. In this case, it is easy to check that

$$
\frac{\partial \pi_{\text {in }}(u, q)}{\partial q}=-(c-s), \quad \frac{\partial^{2} \pi_{\text {in }}(u, q)}{\partial q^{2}}=0 .
$$

Thus, for any given $u$ such that $u<q, \pi_{\text {in }}(u, q)$ linearly decreases in $q$. Also, for any given $u$ such that $u \geq q$, $\pi_{\text {in }}(u, q)$ is the expected profit for a traditional newsvendor problem with fixed market price $p(u)$, and thus it is concave in $q$. Therefore, for any given $u, \pi_{\text {in }}(u, q)$ is concave in $q$. Considering that $\Pi_{\text {in }}(q)$ is the integration of $\pi_{\text {in }}(u, q)$ over $u$, $\Pi_{\text {in }}(q)$ is concave, and the optimal solution can be uniquely obtained, as shown in the following proposition.

Proposition 3. Consider the in-house production model. (i) The expected profit $\Pi_{i n}(q)$ is concave and (ii) the optimal order quantity, denoted as $q_{i n}^{*}$, is unique and satisfies

$$
\bar{G}\left(q_{i n}^{*}\right)=\frac{c-s}{\int_{q_{i n}^{*}}^{+\infty} p(u) f(u) d u-s \bar{F}\left(q_{i n}^{*}\right)} .
$$

Proposition 3 gives the unique optimal condition for the order quantity for the in-house production case. From (2), (5), and (10), we can see some similarity among $\bar{G}\left(q_{0}^{*}\right)$, $\bar{G}\left(q_{\text {in }}^{*}\right)$, and $\bar{G}\left(q_{\mathrm{pc}}^{*}\right)$. Again, we can establish some relationship between the optimal quantities, as summarized in the following proposition.

Proposition 4. The optimal order quantity for the in-house production case $q_{i n}^{*}$ is no greater than the optimal order quantity for the traditional newsvendor model with fixed market price $p\left(q_{i n}^{*}\right)$; that is,

$$
q_{\text {in }}^{*} \leq q_{0}^{*}\left(p\left(q_{\text {in }}^{*}\right)\right) .
$$

In particular, if the market price is a constant, denoted as $p$, then

$$
q_{i n}^{*} \leq q_{0}^{*}(p) .
$$

Recall that, in the procurement case, we have a similar relation between $q_{\mathrm{pc}}^{*}$ and $q_{0}^{*}\left(p\left(q_{\mathrm{pc}}^{*}\right)\right)$, where the equality can be attained when the market price is a constant (see Proposition 2(ii)). However, in the in-house production case, the equality cannot be attained even if the market price is a constant. This is easy to understand. Compared with the traditional newsvendor model, the in-house production model may need to pay for the quantities more than what was received. Thus, even if there is no uncertainty in the market price, the possible payment for the quantities that are not received avoids the firm ordering as high as that in the traditional model.

We can also compare the optimal quantities between the two cost models.

\section{Proposition 5. $q_{p c}^{*}>q_{i n}^{*}$.}

Proposition 5 says that the optimal order quantity in the in-house production case is no greater than the optimal order quantity in the procurement case. This is consistent with the intuitive belief. In the in-house production case, the firm always needs to pay for quantity ordered, even if it is smaller than the quantity received. However, in the procurement case, the firm only needs to pay for the quantity received. Thus, the firm facing in-house production cost has no incentive to order as high as the one facing procurement case.

Remark 6. Proposition 5 is valid because we let the unit production cost $c$ be the same in the two cases. Consider the case where the unit production costs for the two cases are different. Let $c_{\mathrm{pc}}$ and $c_{\mathrm{in}}$ be the unit production costs in the procurement case and the in-house production case, respectively. If $c_{\text {in }} \geq c_{\mathrm{pc}}$, it is easy to check that $\bar{G}\left(q_{\text {in }}^{*}\right) \geq$ $\bar{G}\left(q_{\mathrm{pc}}^{*}\right)$. This implies that $q_{\mathrm{pc}}^{*} \geq q_{\mathrm{in}}^{*}$ and that Proposition 5 is still valid. However, if $c_{\text {in }}<c_{\mathrm{pc}}$, Proposition 5 may not be valid.

\section{Computational Study}

We now conduct the computational study to investigate the sensitivity of the optimal solution versus the distribution of 


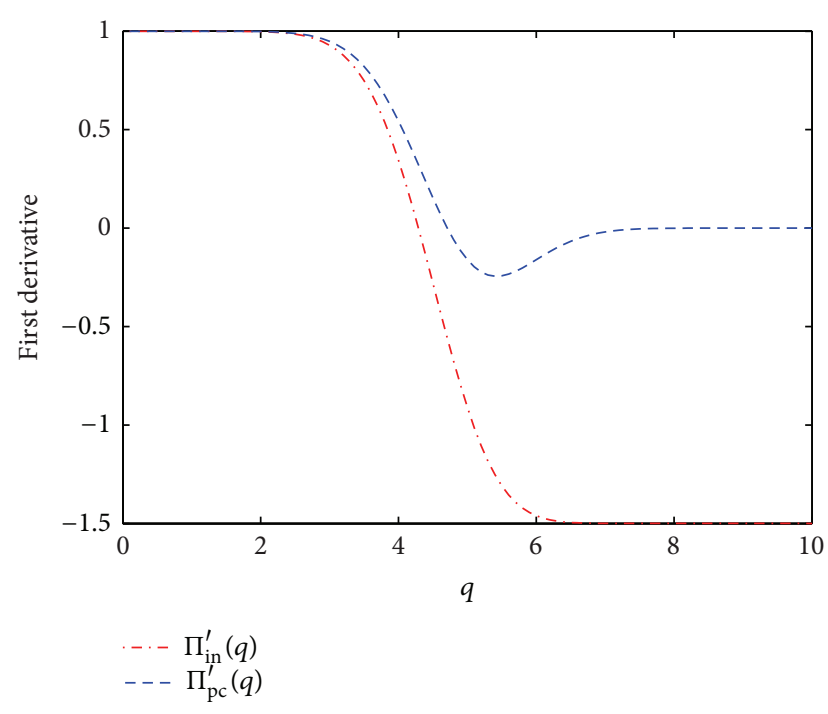

FIgURE 1: The first derivative of the expected profit.

the random capacity. We adopt the settings from $\mathrm{Wu}$ et al. [28]: let the market demand and the random capacity both follow a normal distribution with mean $\mu=5$ and standard deviation $\sigma=1$, let the unit ordering cost be $c=2$, and let the salvage value be $s=0.2$. In our computational experiment, we will set an error tolerance level $1 \times 10^{-4}$. With this tolerance level, we can restrict our attention to the capacity in the interval $[0,10]$, because the probability of the capacity lying out of this interval is less than $1 \times 10^{-4}$. In Wu et al. [28], they consider fixed market price 3 . In our computational study, we consider a linear price function: $p(u)=a u+b$, where $a$ and $b$ are the coefficients. We determine $a$ and $b$ by requiring that (i) the expectation of the market price is 3 , that is,

$$
\mathbb{E} p(u)=a \mathbb{E} u+b=5 a+b=3,
$$

and (ii) the lowest price equals $c=2$ and is attained at the upper bound $u=10$ such that we always have $p(u) \geq c$ for $u \in[0,10]$; that is,

$$
p(10)=10 a+b=2 .
$$

Solving the equation set (13)-(14), we obtain $a=-0.2$ and $b=4$. Therefore, we use the price function

$$
p(u)=4-0.2 u
$$

in our experiment.

Under this setting, the first derivatives of the expected profit in the two cases are depicted in Figure 1. We can see that $\Pi_{\text {in }}^{\prime}(q)$ decreases, and $\Pi_{\mathrm{pc}}^{\prime}(q)$ first decreases, then increases, and never turns to be positive. There is only one zero root of $\Pi_{\mathrm{in}}^{\prime}(q)\left(\Pi_{\mathrm{pc}}^{\prime}(q)\right)$. This is consistent with the results in Propositions 2 and 4.

We then conduct the sensitivity analysis of the optimal solutions versus the random demand and the random

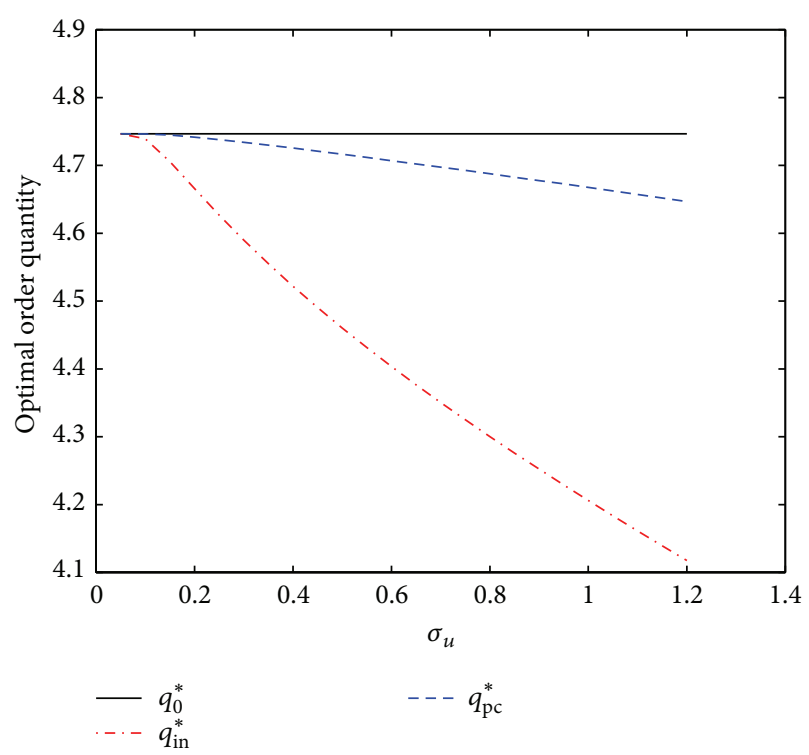

FIgURe 2: The optimal order quantities versus $\sigma_{u}$.

capacity. We first consider the optimal solution versus the random capacity $u$. Let the demand distribution fix and follow a normal distribution with a mean 5 and a deviation 1. Regarding the distribution of the random capacity, we consider a normal distribution with a mean 5 but with different standard deviations, denoted as $\sigma_{u}$. It is easy to check that when $\sigma_{u} \leq 1.2$, we can still restrict our attention to $u$ such that $u \in[0,10]$ with the tolerance level $1 \times 10^{-4}$. Then, it is still reasonable to use the price function $p(u)=4-0.2 u$. Thus, we let $\sigma_{u}$ vary from 0.05 to 1.2 with a step of 0.05 . For each value of $\sigma_{u}$, we calculate the optimal order quantities for the two cases as well as the optimal order quantity for the traditional newsvendor model with market price equaling $\mathbb{E} p(u)=3$. These optimal quantities are depicted in Figure 2. The optimal order quantity for the traditional newsvendor model is a constant, because the random capacity is not considered and the demand distribution does not change. We can see that the optimal quantity for the in-house production case is smaller than that for the procurement case, just as shown in Proposition 5. An important observation from Figure 2 is that the optimal order quantities for both of the two cases decrease as $\sigma_{u}$ increases. This means that when the random capacity has greater deviation, the firm has the incentive to order less. Another observation is that the decrease of the optimal quantity for the in-house production case is sharper than the decrease of optimal quantity for the procurement case. This is because, in the in-house production case, the firm has to pay for the quantity ordered. Compared with the procurement case, the in-house production case's expected profit can be affected by the random capacity to a greater extent. Thus, for a firm where the in-house production model is adopted, the manager will be more careful when the capacity has greater deviation.

We next consider the optimal solution versus the random demand $d$. Let the capacity distribution fix and follow 


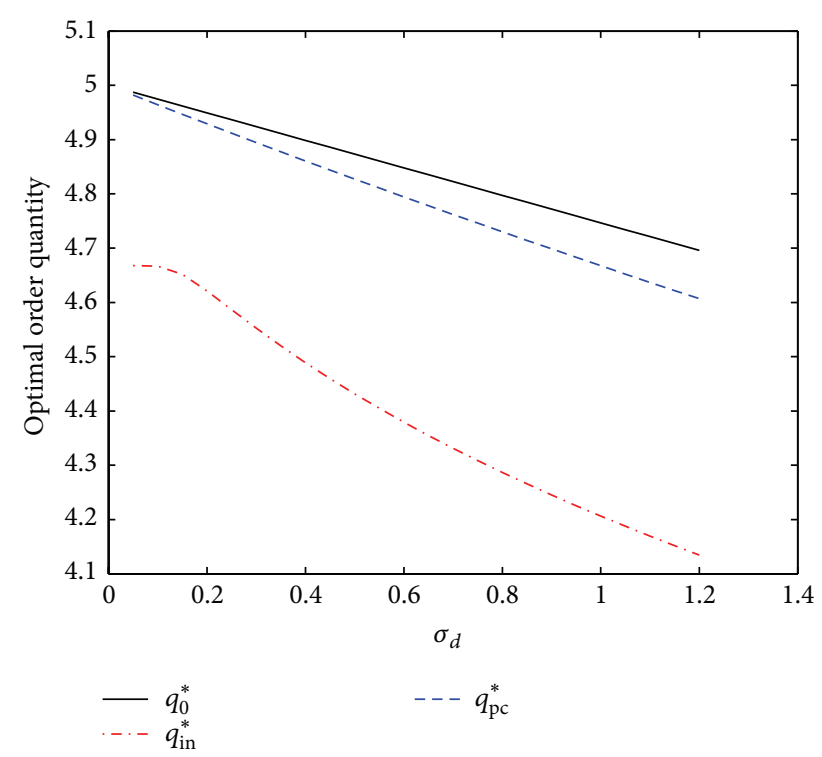

FIgURE 3: The optimal order quantities versus $\sigma_{d}$.

a normal distribution with a mean 5 and a deviation 1, and let the distribution of the demand distribution vary: the mean value is fixed at 5 and the standard deviation, denoted as $\sigma_{d}$, varies from 0.05 to 1.2 with a step of 0.05 . Under this setting, we can still restrict our attention to $u$ such that $u \in[0,10]$ with the tolerance level $1 \times 10^{-4}$. Similarly, we use the price function $p(u)=4-0.2 u$. For each value of $\sigma_{d}$, we calculate the optimal order quantities for the two cases as well as the optimal order quantity for the traditional newsvendor model with market price equaling $\mathbb{E} p(u)=$ 3. These optimal quantities are depicted in Figure 3. We observe that the optimal order quantities in the three cases all decrease when the standard deviation increases, the optimal order quantity in the in-house production case is always the smallest, and the optimal order quantity in the traditional newsvendor model is always the greatest. We also observe that, similar to the case depicted in Figure 2, the optimal order quantity in the in-house production case decreases sharper than that in the procurement case or the traditional newsvendor case. Therefore, for a firm adopting the in-house production purchasing cost structure, the manager will be more careful about the volatility of the demand and the capacity.

\section{Conclusion}

In this paper, we consider a newsvendor modeled product system with random capacity and capacity-dependent market price. Two production cost structures are considered: the procurement cost structure where the firm pays for the received quantity and the in-house production cost structure where the firm pays for the input quantity. The market price depends on the random capacity, and we consider a general form only requiring that the price decreases in the capacity. We show that the optimal quantity is unique and specify the optimal conditions under both cost structures. Comparing the optimal quantities, we find that, in each case, the optimal order quantity is no greater than the optimal quantity in the traditional newsvendor model with a fixed market price. We also find that the optimal solution for the procurement case is no smaller than that for the in-house production case. Numerical studies are also conducted to investigate the sensitivity of the optimal solution versus the distribution of the random capacity. We observe that the optimal quantity decreases in the deviation of the random capacity/demand in both of the two cases and that the optimal quantity of the in-house production case is more sensitive in the deviation of the random capacity/demand. The managers will be more careful about the volatility of the random capacity/demand if the system is adopting an in-house production purchasing cost structure.

One limitation of this paper is that we consider a priceindependent demand process. The price-dependent demand has been widely considered in the literature (see, e.g., $\mathrm{Xu}$ and $\mathrm{Lu}$ [37]) but not in the scope of this work. Besides, what we considered in this paper is a risk-neutral decision maker who maximizes the expected profit. The other riskaverse objectives such as $\mathrm{VaR}$ and $\mathrm{CVaR}$ may be taken as another possible research direction (see, e.g., Shen et al. [40]). Moreover, we assume that the random capacity and the demand are two independent processes in this paper. It might be more practical if we allow the two random variables to be dependent. However, this would greatly increase the difficulty of solving the problem. We take it as an interesting future research topic.

\section{Appendix}

Proof of Proposition 1. Consider (3). We have the following derivatives:

$$
\begin{gathered}
\frac{\partial \pi_{\mathrm{pc}}(u, q)}{\partial q}=1_{\{u \geq q\}}[(p(u)-s) \bar{G}(q)-(c-s)] \\
\frac{\partial^{2} \pi_{\mathrm{pc}}(u, q)}{\partial q^{2}}=-1_{\{u \geq q\}}(p(u)-s) g(q) .
\end{gathered}
$$

Next, we consider the first- and second-order derivatives of $\Pi_{\mathrm{pc}}(q)$. Note that, from the first case of (3), we have $\pi_{\mathrm{pc}}(u, q)=\pi_{\mathrm{pc}}(u, u)$ for any $u<q$. Thus, from (4), we have

$$
\begin{aligned}
& \frac{\partial \Pi_{\mathrm{pc}}(q)}{\partial q} \\
& \quad=\frac{\partial}{\partial q} \int_{0}^{q} \pi_{\mathrm{pc}}(u, u) f(u) d u \\
& \quad+\frac{\partial}{\partial q} \int_{q}^{+\infty} \pi_{\mathrm{pc}}(u, q) f(u) d u \\
& =\pi_{\mathrm{pc}}(q, q) f(q)
\end{aligned}
$$




$$
\begin{aligned}
& +\int_{q}^{+\infty} \frac{\partial \pi_{\mathrm{pc}}(u, q)}{\partial q} f(u) d u-\pi_{\mathrm{pc}}(q, q) f(q) \\
= & \int_{q}^{+\infty} \frac{\partial \pi_{\mathrm{pc}}(u, q)}{\partial q} f(u) d u,
\end{aligned}
$$

which implies that

$$
\frac{\partial^{2} \Pi_{\mathrm{pc}}(q)}{\partial q^{2}}=\int_{q}^{+\infty} \frac{\partial^{2} \pi_{\mathrm{pc}}(u, q)}{\partial q^{2}} f(u) d u-\frac{\partial \pi_{\mathrm{pc}}(q, q)}{\partial q} f(q) .
$$

In the right-hand side of the above equation, we have $\int_{q}^{+\infty}\left(\partial^{2} \pi_{\mathrm{pc}}(u, q) / \partial q^{2}\right) f(u) d \leq 0$ (see (A.2), where $(p(u)-$ s) $g(q) \geq 0)$. However, it is not easy to check if the second term $\partial \pi_{\mathrm{pc}}(q, q) / \partial q$ is positive or negative. Thus, we next restrict our attention to check the sign of the second-order derivative at $\bar{q}$ where $\left.\left(\partial \Pi_{p c}(q) / \partial q\right)\right|_{q=\bar{q}}=0$.

Recall that $p(u)$ decreases in $u$. From (A.2), $\partial \pi_{\mathrm{pc}}(u, q) / \partial q$ decreases in $u$ over the interval $u \in[q,+\infty]$. Thus, if

$$
\left.\frac{\partial \Pi_{\mathrm{pc}}(q)}{\partial q}\right|_{q=\bar{q}}=\left.\int_{q}^{+\infty} \frac{\partial \pi_{\mathrm{pc}}(u, q)}{\partial q} f(u) d u\right|_{q=\bar{q}}=0,
$$

then we must have

$$
\left.\frac{\partial \pi_{\mathrm{pc}}(q, q)}{\partial q}\right|_{q=\bar{q}} \geq 0
$$

Otherwise,

$$
\frac{\partial \pi_{\mathrm{pc}}(u, q)}{\partial q} \leq 0 \quad \text { for any } u \geq \bar{q}
$$

which implies that

$$
\left.\frac{\partial \Pi_{\mathrm{pc}}(q)}{\partial q}\right|_{q=\bar{q}}=\left.\int_{q}^{+\infty} \frac{\partial \pi_{\mathrm{pc}}(u, q)}{\partial q} f(u) d u\right|_{q=\bar{q}} \leq 0 .
$$

This is a contradiction. Hence, we have

$$
\left.\frac{\partial \pi_{\mathrm{pc}}(q, q)}{\partial q}\right|_{\left(\partial \Pi_{\mathrm{pc}}(q) / \partial q\right)=0} \geq 0 .
$$

Considering this, together with (A.4) and the fact that $\int_{q}^{+\infty}\left(\partial^{2} \pi_{\mathrm{pc}}(u, q) / \partial q^{2}\right) f(u) d<0$, we have

$$
\begin{aligned}
& \left.\frac{\partial^{2} \Pi_{\mathrm{pc}}(u, q)}{\partial q^{2}}\right|_{\left(\partial \Pi_{r}(u, q) / \partial q\right)=0} \\
& =\left[\int_{q}^{+\infty} \frac{\partial^{2} \pi_{\mathrm{pc}}(u, q)}{\partial q^{2}} f(u) d u\right. \\
& \left.\quad-\frac{\partial \pi_{\mathrm{pc}}(q, q)}{\partial q} f(q)\right]\left.\right|_{\left(\partial \Pi_{\mathrm{pc}}(u, q) / \partial q\right)=0}<0 .
\end{aligned}
$$

Therefore, the expected profit is quasi-concave, and the optimal order quantity is the unique zero root of the firstorder derivative.

Regarding the first-order derivative, from (A.1) and (A.3), we have

$$
\begin{aligned}
& \frac{\partial \Pi_{\mathrm{pc}}(q)}{\partial q} \\
& \quad=\int_{q}^{+\infty} \frac{\partial \pi_{\mathrm{pc}}(u, q)}{\partial q} f(u) d u \\
& \quad=\int_{q}^{+\infty}[(p(u)-s) \bar{G}(q)-(c-s)] f(u) d u \\
& \quad=\left[\int_{q}^{+\infty} p(u) f(u) d u-s \bar{F}(q)\right] \bar{G}(q)-(c-s) \bar{F}(q)
\end{aligned}
$$

Thus, the optimal order quantity $q_{\mathrm{pc}}^{*}$ satisfies

$$
\bar{G}\left(q_{\mathrm{pc}}^{*}\right)=\frac{(c-s) \bar{F}\left(q_{\mathrm{pc}}^{*}\right)}{\int_{q_{\mathrm{pc}}^{*}}^{+\infty} p(u) f(u) d u-s \bar{F}\left(q_{\mathrm{pc}}^{*}\right)} .
$$

Proof of Proposition 2. Recall that $p(u)$ decreases in $u$. From (5)

$$
\begin{aligned}
\bar{G}\left(q_{\mathrm{pc}}^{*}\right) & =\frac{(c-s) \bar{F}\left(q_{\mathrm{pc}}^{*}\right)}{\int_{q_{\mathrm{pc}}^{*}}^{+\infty} p(u) f(u) d u-s \bar{F}\left(q_{\mathrm{pc}}^{*}\right)} \\
& \geq \frac{(c-s) \bar{F}\left(q_{\mathrm{pc}}^{*}\right)}{p\left(q_{\mathrm{pc}}^{*}\right) \int_{q_{\mathrm{pc}}^{*}}^{+\infty} f(u) d u-s \bar{F}\left(q_{\mathrm{pc}}^{*}\right)} \\
& =\frac{(c-s) \bar{F}\left(q_{\mathrm{pc}}^{*}\right)}{\left(p\left(q_{\mathrm{pc}}^{*}\right)-s\right) \bar{F}\left(q_{\mathrm{pc}}^{*}\right)} \\
& =\frac{c-s}{p\left(q_{\mathrm{pc}}^{*}\right)-s},
\end{aligned}
$$


where the equality of the inequality holds if and only if $p(u)$ is a constant. Note also that the optimal order quantity in the traditional newsvendor model satisfies (2). Thus,

$$
\bar{G}\left(q_{0}^{*}\left(q_{\mathrm{pc}}^{*}\right)\right)=\frac{c-s}{p\left(q_{\mathrm{pc}}^{*}\right)-s} .
$$

Compare $\bar{G}\left(q_{\mathrm{pc}}^{*}\right)$ and $\bar{G}\left(q_{0}^{*}\left(q_{\mathrm{pc}}^{*}\right)\right)$ given above. We conclude that $q_{\mathrm{pc}}^{*} \leq q_{0}^{*}\left(p\left(q_{\mathrm{pc}}^{*}\right)\right)$, and the equality holds if and only if $p(u)$ is a constant.

Proof of Proposition 3. (i) From (7), we have the first- and second-order derivatives of $\pi_{\text {in }}(u, q)$ with respect to $q$ as follows:

$$
\begin{gathered}
\frac{\partial \pi_{\text {in }}(u, q)}{\partial q}=1_{\{u \geq q\}}(p(u)-s) \bar{G}(q)-(c-s), \\
\frac{\partial^{2} \pi_{\text {in }}(u, q)}{\partial q^{2}}=-1_{\{u \geq q\}}(p(u)-s) g(q) \leq 0 .
\end{gathered}
$$

From the last equation, we have

$$
\begin{aligned}
\frac{\partial^{2} \Pi_{\text {in }}(q)}{\partial q^{2}} & =\frac{\partial^{2} \int_{0}^{\infty} \pi_{\text {in }}(u, q) d u}{\partial q^{2}} \\
& =\int_{0}^{+\infty} \frac{\partial^{2} \pi_{\text {in }}(u, q)}{\partial q^{2}} f(u) d u \\
& =-\int_{q}^{+\infty}(p(u)-s) g(q) f(u) d u \leq 0,
\end{aligned}
$$

where the last inequality holds because $p(u)-s \geq 0$ for any $u$. Thus, the expected profit is concave in $q$.

(ii) Consider the first derivative of $\Pi(q)$ over $q$. We have

$$
\begin{aligned}
& \frac{\partial \Pi_{\text {in }}(q)}{\partial q} \\
& =\frac{\partial}{\partial q} \int_{0}^{+\infty} \pi_{\text {in }}(u, q) f(u) d u \\
& =\frac{\partial}{\partial q} \int_{0}^{q} \pi_{\text {in }}(u, q) f(u) d u \\
& \quad+\frac{\partial}{\partial q} \int_{q}^{+\infty} \pi_{\text {in }}(u, q) f(u) d u \\
& =\int_{0}^{q} \frac{\partial \pi_{\text {in }}(u, q)}{\partial q} f(u) d u+\pi_{\text {in }}(q, q) f(q) \\
& \quad+\int_{q}^{+\infty} \frac{\partial \pi_{\text {in }}(u, q)}{\partial q} f(u) d u-\pi_{\text {in }}(q, q) f(q) \\
& =\int_{0}^{q} \frac{\partial \pi_{\text {in }}(u, q)}{\partial q} f(u) d u+\int_{q}^{+\infty} \frac{\partial \pi_{\text {in }}(u, q)}{\partial q} f(u) d u
\end{aligned}
$$

$$
\begin{aligned}
& =-(c-s)+\bar{G}(q) \int_{q}^{+\infty}(p(u)-s) f(u) d u \\
& =s-c+\bar{G}(q)\left[\int_{q}^{+\infty} p(u) f(u) d u-s \bar{F}(q)\right] .
\end{aligned}
$$

The optimal order quantity $q^{*}$ satisfies

$$
\left.\frac{\partial \Pi_{\text {in }}(q)}{\partial q}\right|_{q=q^{*}}=0,
$$

or, equivalently, we have

$$
\bar{G}\left(q^{*}\right)=\frac{c-s}{\int_{q^{*}}^{+\infty} p(u) f(u) d u-s \bar{F}\left(q^{*}\right)} .
$$

Proof of Proposition 4. Consider the optimal order quantity in the in-house production model. From (10), we have

$$
\begin{aligned}
\bar{G} & \left(q_{\text {in }}^{*}\right) \\
& =\frac{c-s}{\int_{q_{\text {in }}^{*}}^{+\infty} p(u) f(u) d u-s \bar{F}\left(q_{\text {in }}^{*}\right)} \\
& \geq \frac{c-s}{\int_{q_{\text {in }}^{*}}^{+\infty} p\left(q_{\text {in }}^{*}\right) f(u) d u-s \bar{F}\left(q_{\text {in }}^{*}\right)} \\
& =\frac{c-s}{\left(p\left(q_{\text {in }}^{*}\right)-s\right) \bar{F}\left(q_{\text {in }}^{*}\right)} \geq \frac{c-s}{\left(p\left(q_{\text {in }}^{*}\right)-s\right)},
\end{aligned}
$$

where the first inequality holds because $p(u)$ is nonincreasing in $u$ and the second inequality holds because $\bar{F}\left(q_{\text {in }}^{*}\right) \leq 1$. Next, we consider the traditional newsvendor model. From (2), we have

$$
\bar{G}\left(q_{0}^{*}\left(p\left(q_{\text {in }}^{*}\right)\right)\right)=\frac{c-s}{p\left(q_{\text {in }}^{*}\right)-s} .
$$

Comparing the above two equations, we have

$$
\bar{G}\left(q_{\text {in }}^{*}\right) \geq \bar{G}\left(q_{0}^{*}\left(p\left(q_{\text {in }}^{*}\right)\right)\right),
$$

which implies that $q_{\text {in }}^{*} \leq q_{0}^{*}\left(p\left(q_{\text {in }}^{*}\right)\right)$.

Proof of Proposition 5. Compare (10) and (5); it is easy to see that

$$
\bar{G}\left(q_{\mathrm{pc}}^{*}\right) \leq \bar{G}\left(q_{\mathrm{in}}^{*}\right) .
$$

\section{Notations}

D: $\quad$ Demand variable with $G(\cdot)$ and $g(\cdot)$ being its $c d f$ and $p d f$, respectively

$u$ : $\quad$ Random supply capacity with $F(\cdot)$ and $f(\cdot)$ being its $c d f$ and $p d f$, respectively

$p(u)$ : Unit selling price, which depends on the supply capacity

$c$ : Unit production cost

$s$ : Unit salvage value. 


\section{Conflict of Interests}

The authors declare that there is no conflict of interests regarding the publication of this paper.

\section{Acknowledgments}

The authors would like to thank the Academic Editor and three anonymous referees for the helpful suggestions and comments. This research was supported in part by the "Shanghai Pujiang Program (14PJ1400200)." It was also supported in part by the "Fundamental Research Funds for the Central Universities."

\section{References}

[1] M. Khouja, "The single-period (news-vendor) problem: literature review and suggestions for future research," Omega, vol. 27, no. 5, pp. 537-553, 1999.

[2] Y. Qin, R. Wang, A. J. Vakharia, Y. Chen, and M. M. H. Seref, "The newsvendor problem: review and directions for future research," European Journal of Operational Research, vol. 213, no. 2, pp. 361-374, 2011.

[3] M. M. Tajbakhsh, S. Zolfaghari, and G. G. Lee, "Supply uncertainty and diversification: a review," in Trends in Supply Chain Design and Management: Technologies and Methodologies, $\mathrm{H}$. Jung, B. Jeong, and F. F. Chen, Eds., pp. 345-368, Springer, London, UK, 2007.

[4] C. A. Yano and H. L. Lee, "Lot sizing with random yields: a review," Operations Research, vol. 43, no. 2, pp. 311-334, 1995.

[5] L. V. Snyder, Z. P. Atan, Y. Peng, A. J. Rong, B. Schmitt, and B. Sinsoysal, "OR/MS models for supply chain disruptions: a review," Tech. Rep., Lehigh University, Bethlehem, PA, USA, 2014.

[6] H. Gurnani, R. Akella, and J. Lehoczky, "Supply management in assembly systems with random yield and random demand," IIE Transactions, vol. 32, no. 8, pp. 701-714, 2000.

[7] B. Tomlin and Y. Wang, "On the value of mix flexibility and dual sourcing in unreliable newsvendor networks," Manufacturing \& Service Operations Management, vol. 7, no. 1, pp. 37-57, 2005.

[8] M. Dada, N. C. Petruzzi, and L. B. Schwarz, "A newsvendor's procurement problem when suppliers are unreliable," Manufacturing \& Service Operations Management, vol. 9, no. 1, pp. 9-32, 2007.

[9] B. C. Giri, "Managing inventory with two suppliers under yield uncertainty and risk aversion," International Journal of Production Economics, vol. 133, no. 1, pp. 80-85, 2011.

[10] S. Y. Tang and P. Kouvelis, "Supplier diversification strategies in the presence of yield uncertainty and buyer competition," Manufacturing and Service Operations Management, vol. 13, no. 4, pp. 439-451, 2011.

[11] X. Chao, X. Gong, and S. Zheng, "Optimal pricing and inventory policies with reliable and random-yield suppliers: characterization and comparison," Annals of Operations Research, 2014.

[12] R. R. Meyer, M. H. Rothkopf, and S. A. Smith, "Reliability and inventory in a production-storage system," Management Science, vol. 25, no. 8, pp. 799-807, 1979.

[13] T. Bielecki and P. R. Kumar, "Optimality of zero-inventory policies for unreliable manufacturing systems," Operations Research, vol. 36, no. 4, pp. 532-541, 1988.
[14] M. Parlar and D. Berkin, "Future supply uncertainty in EOQ models," Naval Research Logistics, vol. 38, no. 1, pp. 107-121, 1991.

[15] M. Parlar and D. Perry, "Analysis of a $(Q, r, T)$ inventory policy with deterministic and random yields when future supply is uncertain," European Journal of Operational Research, vol. 84, no. 2, pp. 431-443, 1995.

[16] M. Parlar, "Continuous-review inventory problem with random supply interruptions," European Journal of Operational Research, vol. 99, no. 2, pp. 366-385, 1997.

[17] K. Moinzadeh and P. Aggarwal, "Analysis of a production/inventory system subject to random disruptions," Management Science, vol. 43, no. 11, pp. 1577-1588, 1997.

[18] A. Arreola-Risa and G. A. DeCroix, "Inventory management under random supply disruptions and partial backorders," Naval Research Logistics, vol. 45, no. 7, pp. 687-703, 1998.

[19] B. Tomlin, "On the value of mitigation and contingency strategies for managing supply chain disruption risks," Management Science, vol. 52, no. 5, pp. 639-657, 2006.

[20] S. Chopra, G. Reinhardt, and U. Mohan, "The importance of decoupling recurrent and disruption risks in a supply chain," Naval Research Logistics, vol. 54, no. 5, pp. 544-555, 2007.

[21] J. Li, S. Wang, and T. C. E. Cheng, "Competition and cooperation in a single-retailer two-supplier supply chain with supply disruption," International Journal of Production Economics, vol. 124, no. 1, pp. 137-150, 2010.

[22] G. Gupta, B. He, and S. P. Sethi, "Contingent sourcing under supply disruption and competition," International Journal of Production Research, 2014.

[23] P. Ray and M. Jenamani, "Sourcing decision under disruption risk with supply and demand uncertainty: a newsvendor approach," Annals of Operations Research, 2014.

[24] K. J. Arrow, S. Karlin, and H. Scarf, Studies in the Mathematic Theory of Inventory and Production, Stanford University Press, Stanford, Calif, USA, 1958.

[25] F. W. Ciarallo, R. Akella, and T. E. Morton, "A periodic review, production planning model with uncertain capacity and uncertain demand-optimality of extended myopic policies," Management Science, vol. 40, no. 3, pp. 320-332, 1994.

[26] Y. A. Wang and Y. Gerchak, "Periodic review production models with variable capacity, random yield, and uncertain demand," Management Science, vol. 42, no. 1, pp. 130-137, 1996.

[27] H. K. Okyay, F. Karaesmen, and S. Özekici, "Newsvendor models with dependent random supply and demand," Optimization Letters, vol. 8, no. 3, pp. 983-999, 2014.

[28] M. Wu, S. X. Zhu, and R. H. Teunter, "The risk-averse newsvendor problem with random capacity," European Journal of Operational Research, vol. 231, no. 2, pp. 328-336, 2013.

[29] R. Bollapragada, U. S. Rao, and J. Zhang, "Managing inventory and supply performance in assembly systems with random supply capacity and demand," Management Science, vol. 50, no. 12, pp. 1729-1743, 2004.

[30] A. S. Erdem, M. M. Fadiloglu, and S. Özekici, "An EOQ model with multiple suppliers and random capacity," Naval Research Logistics, vol. 53, no. 1, pp. 101-114, 2006.

[31] D. B. Khang and O. Fujiwara, "Optimality of myopic ordering policies for inventory model with stochastic supply," Operations Research, vol. 48, no. 1, pp. 181-184, 2000.

[32] P. Kouvelis and J. M. Milner, "Supply chain capacity and outsourcing decisions: the dynamic interplay of demand and supply uncertainty," IIE Transactions, vol. 34, no. 8, pp. 717-728, 2002. 
[33] R. Bollapragada, U. S. Rao, and J. Zhang, "Managing two-stage serial inventory systems under demand and supply uncertainty and customer service level requirements," IIE Transactions, vol. 36, no. 1, pp. 73-85, 2004.

[34] B. Kazaz, "Production planning under yield and demand uncertainty with yield-dependent cost and price," Manufacturing \& Service Operations Management, vol. 6, no. 3, pp. 209-224, 2004.

[35] B. Kazaz and S. Webster, "The impact of yield-dependent trading costs on pricing and production planning under supply uncertainty," Manufacturing \& Service Operations Management, vol. 13, no. 3, pp. 404-417, 2011.

[36] C. S. Tang and R. Yin, "Responsive pricing under supply uncertainty," European Journal of Operational Research, vol. 182, no. 1, pp. 239-255, 2007.

[37] M. Xu and Y. Lu, "The effect of supply uncertainty in pricesetting newsvendor models," European Journal of Operational Research, vol. 227, no. 3, pp. 423-433, 2013.

[38] Q. Li and S. Zheng, "Joint inventory replenishment and pricing control for systems with uncertain yield and demand," Operations Research, vol. 54, no. 4, pp. 696-705, 2006.

[39] P. H. Zipkin, Foundations of Inventory Management, McGrawHill, Boston, Mass, USA, 2000.

[40] B. Shen, T.-M. Choi, Y. Wang, and C. K. Y. Lo, “The coordination of fashion supply chains with a risk-averse supplier under the markdown money policy," IEEE Transactions on Systems, Man, and Cybernetics Part A:Systems and Humans, vol. 43, no. 2, pp. 266-276, 2013. 


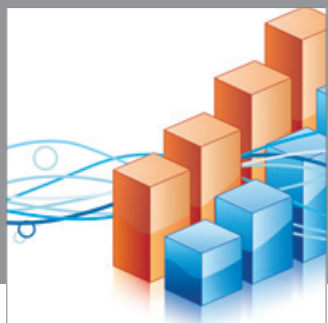

Advances in

Operations Research

mansans

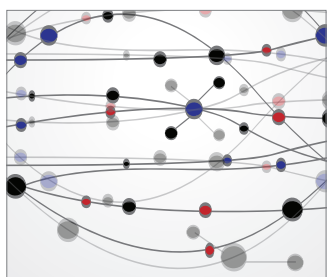

The Scientific World Journal
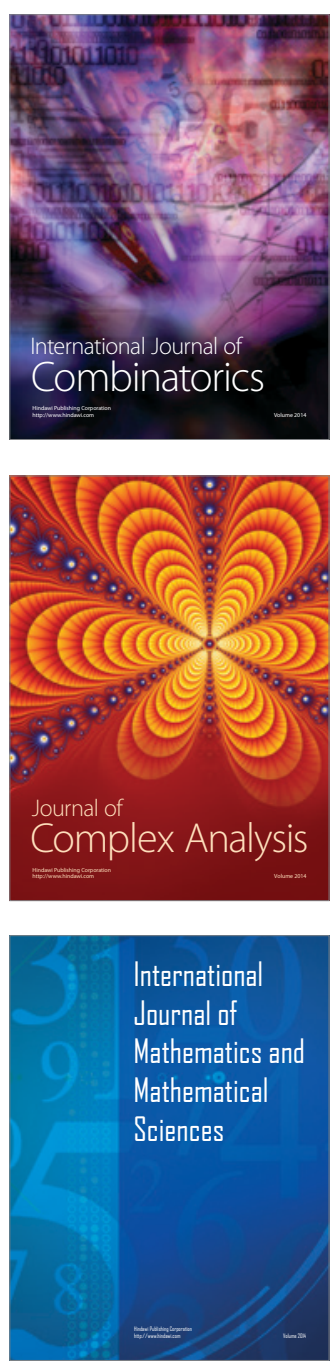
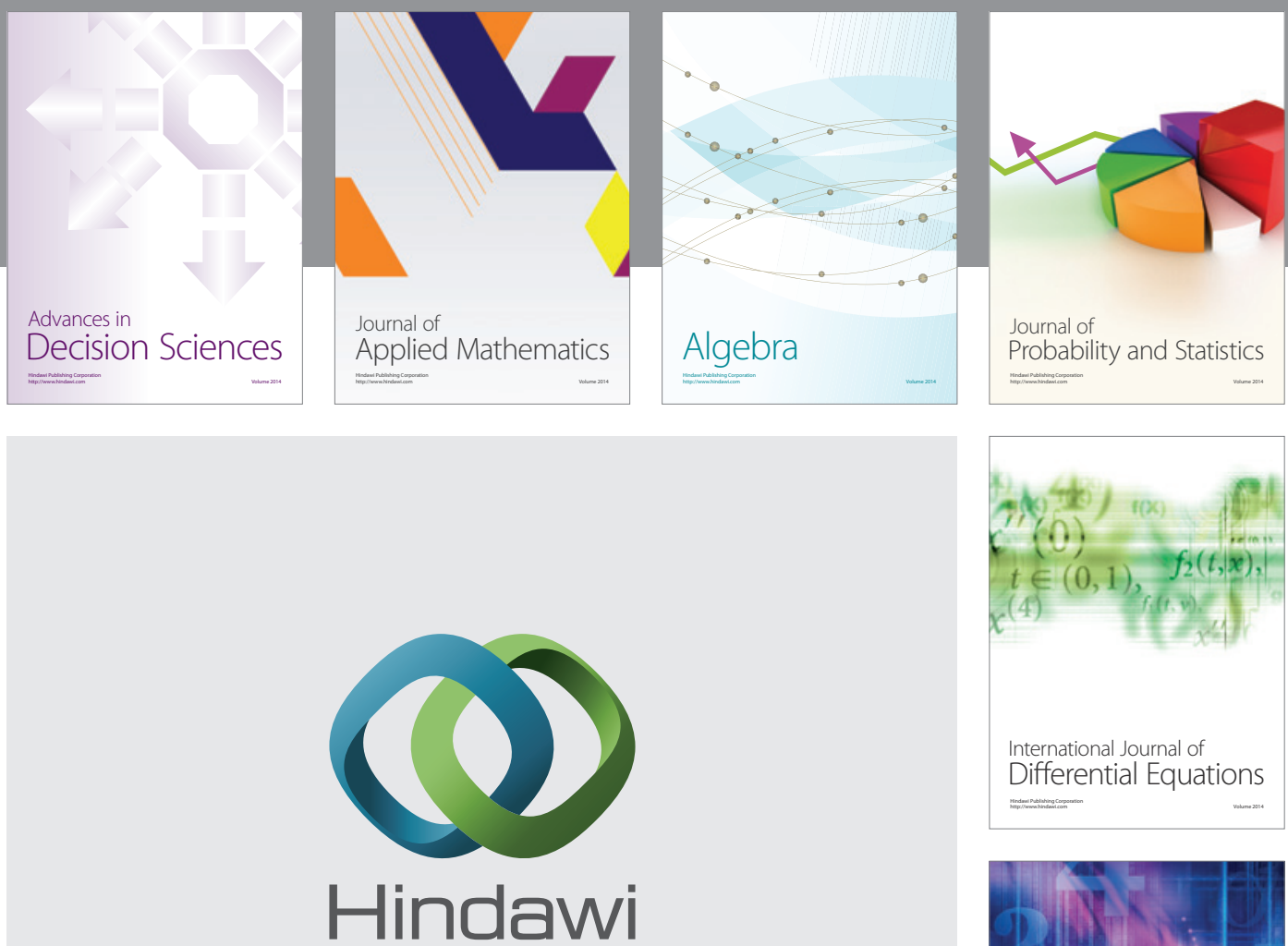

Submit your manuscripts at http://www.hindawi.com
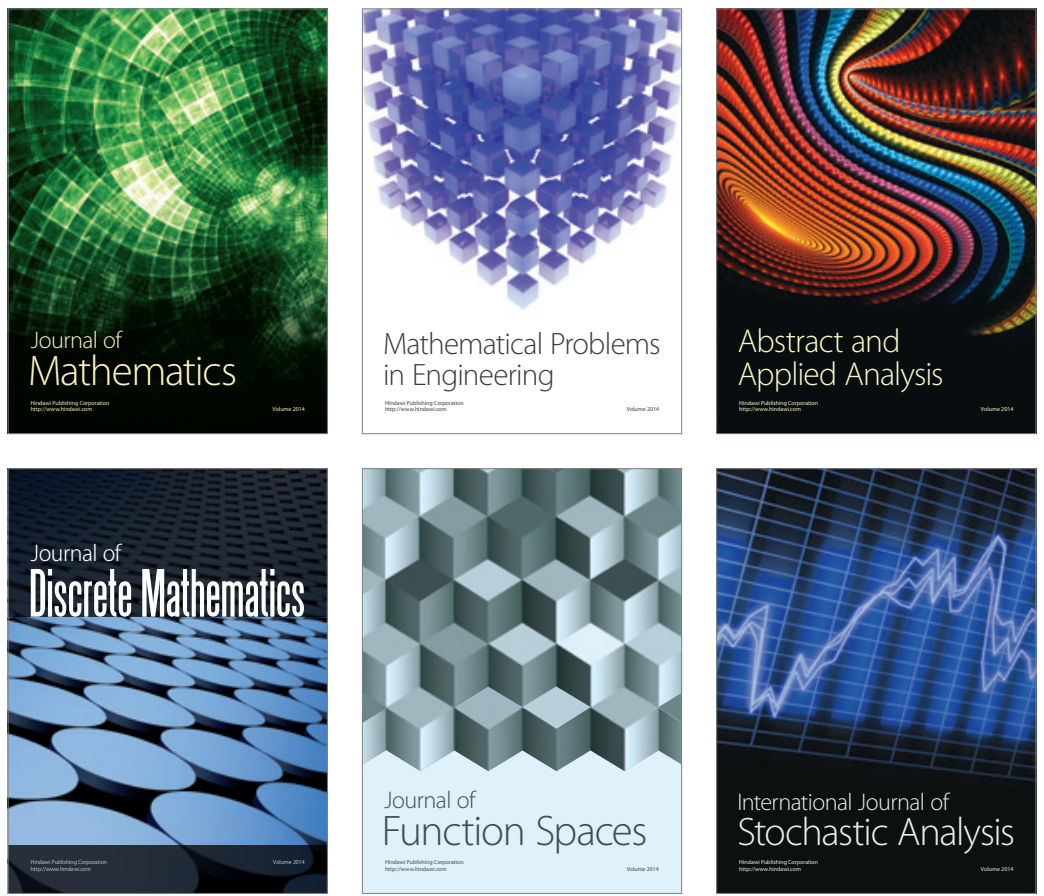

Journal of

Function Spaces

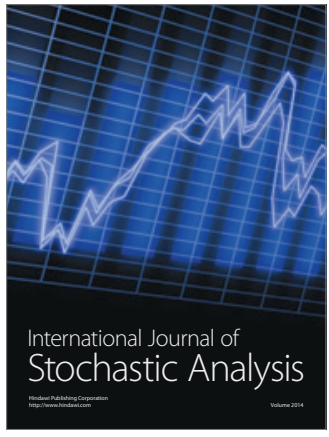

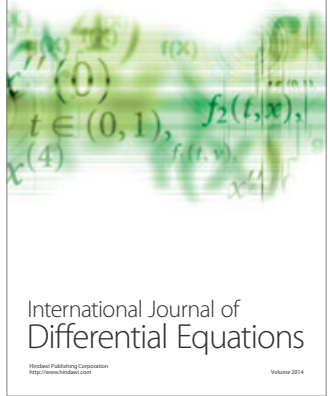
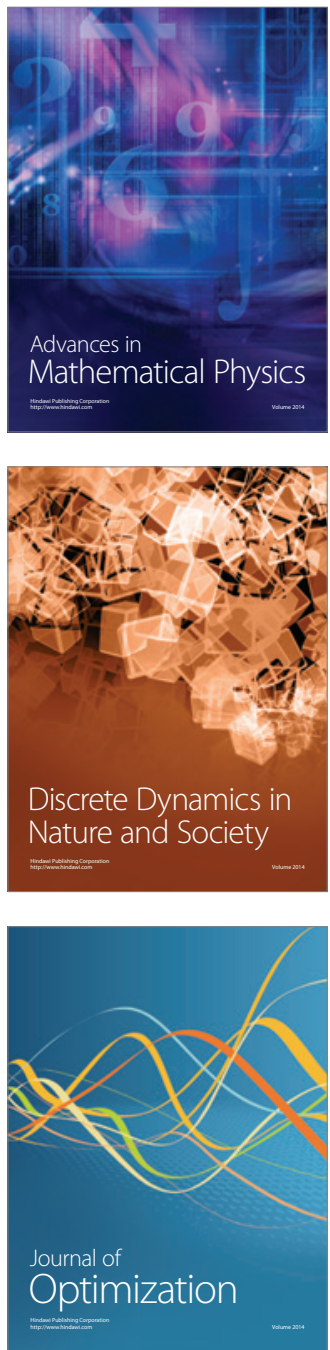